\title{
Correspondence
}

\section{Roasting Roast Breadfruit Psychosis}

Sir: Hickling \& Hutchinson (Psychiatric Bulletin. March 1999, 23, 132-134) play fast and furious with the generally accepted deleterious effect of racism and prejudice on the mental health of the ethnic minority groups in Britain (Littlewood \& Lipsedge, 1989). The epistemological basis for their coinage of 'Roast Breadfruit Syndrome/ Psychosis' does not stand up to serious scrutiny. Some of the fundamental flaws in their argument have been well flagged by the invited commentators. Nevertheless, it is worthwhile to raise other issues that were not given an airing. A significant number of Black immigrants of West African origin, for example Nigerians, also come into contact with psychiatric services in south-east London where one of the authors works. Analysis of the prevalence of severe mental illness among this group is essential to the corroboration of their argument. For it can be argued that recognisably foreign accents and the lack of representation of their cultural traditions in 'Black British' cultural discourse makes this group prone to the same social pressures as African-Caribbean people. Furthermore, certain essential truths about culture were glaringly omitted. Surely it is obvious that no 'culture' can be considered in a vacuum, as cultures are never static and are influenced by other human groups around them constantly adapting and changing. Engaging with minority groups specifically on the basis of, notions of 'culture' is a double-edged sword.

LTTLEWOOD, R. \& LIPSEDGE, M. (1989) Aliens and Alienists: Ethnic Minorities and Psychiatry (2nd edn). London: Unwin Hyman.

OLUROTIMI OJO, Specialist Registrar, DUAL Team, Lewisham \& Guy's Mental Health Trust, 151 Blackfriars Road, London SE1 8EL

Sir: Hickling \& Hutchinson (Psychiatric Bulletin, March 1999, 23, 132-134) present valuable insights on the political and moral importance of 'race' in the construction of 'psychosis' in Black Caribbean people in the UK. The invited commentaries (Psychiatric Bulletin, March 1999. 23. 135-138) seem to miss the message partly because their authors fail to comprehend a fundamental point (made in the paper) namely reluctance in psychiatry to explore the validity of diagnosis itself, preferring instead to dwell in the dubious succour of standardised diagnostic instruments ....

In the nineteenth century, Black slaves who absconded were sometimes diagnosed as suffering from 'drapetomania' which 'manifests itself by an irrestrainable propensity to run away' (Cartwright, 1851). Acting out European (sic) values of liberty was a pathology for them but not for White people. Today too, internalised 'European' values (the meaning of psychotic symptoms as Hickling \& Hutchinson put it) result in problems pathologised as psychosis. If we are to make sense of the high rate at which psychosis is diagnosed among Black people in the UK we have to take on board the sort of insights on how this may come about presented by Hickling \& Hutchinson. We may then see that treatment must be directed at society rather than the individual.

Cartwright, S. A. (1851) Report on the diseases and physical peculiarities of the Negro race. New Orleans Medical and Surgical Joumal, 318, 691-715.

SUMAN FERNANDO, Senior Lecturer in Mental Health. Tizard Centre, University of Kent at Canterbury, Canterbury, Kent CT2 7LZ

Sir: The article by Hickling \& Hutchinson (Psychiatric Bulletin, March 1999, 23, 132-134), was extremely distasteful. The article appeared to have been based purely on speculation, with no evidence offered for the strong views it contained. Very few Black people would fail to be offended by the oft-repeated suggestion that they really would rather be White.

Whatever the inherent causes, and like it or not there are 3.5 million non-Western people in Britain, psychiatrists, entrusted as they are with people's mental well-being, and self-esteem, are obliged, like all other doctors, to carry out their duties in a manner that is free of all bias.

What is perhaps more surprising, is that the respected Psychiatric Bulletin could publish such offensive material (inviting commentaries from African and Asian psychiatrists does not 